\title{
Positive Solutions of a General Discrete Dirichlet Boundary Value Problem
}

\author{
Xinfu Li and Guang Zhang \\ School of Science, Tianjin University of Commerce, Tianjin 300134, China \\ Correspondence should be addressed to Guang Zhang; qd_gzhang@126.com
}

Received 27 October 2015; Accepted 28 December 2015

Academic Editor: Douglas R. Anderson

Copyright ( 92016 X. Li and G. Zhang. This is an open access article distributed under the Creative Commons Attribution License, which permits unrestricted use, distribution, and reproduction in any medium, provided the original work is properly cited.

\begin{abstract}
A steady state equation of the discrete heat diffusion can be obtained by the heat diffusion of particles or the difference method of the elliptic equations. In this paper, the nonexistence, existence, and uniqueness of positive solutions for a general discrete Dirichlet boundary value problem are considered by using the maximum principle, eigenvalue method, sub- and supersolution technique, and monotone method. All obtained results are new and valid on any $n$-dimension finite lattice point domain. To the best of our knowledge, they are better than the results of the corresponding partial differential equations. In particular, the methods of proof are different.
\end{abstract}

\section{Introduction}

In this paper, we consider the discrete Dirichlet boundary value problem

$$
\begin{aligned}
-\Delta x(z) & =f(z, x(z)), \quad z \in \Omega, \\
x(z) & =0, \quad z \in \partial \Omega,
\end{aligned}
$$

where $\Omega \subset Z^{n}$ is a finite domain and $f$ defined on $\Omega \times R$ is a continuous function about the second variable.

To understand the above problem, we need some terminology. Denote the set of integers by $Z$. A lattice point $z=\left(i_{1}, i_{2}, \ldots, i_{n}\right) \in Z^{n}$ is defined as a point with integer coordinates. Two lattice points are said to be neighbors if their Euclidean distance is one. The lattice points $z_{1}, z_{2}, \ldots, z_{m}$ are said to form a path with terminals $z_{1}$ and $z_{m}$ if $z_{1}$ is a neighbor of $z_{2}, z_{2}$ is a neighbor of $z_{3}$, and so forth. A set of lattice points is said to be connected if any two of its points are terminals of a path of points contained in the set. A nonempty connected set of lattice points is called a domain. Given a domain $\Omega$, a lattice point is an exterior boundary point of $\Omega$ if it does not belong to $\Omega$ but has at least one neighbor in $\Omega$. We will denote the set of exterior boundary points of $\Omega$ by $\partial \Omega$, and the set $\Omega \cup \partial \Omega$ of lattice points will be denoted by $\bar{\Omega}$. For a finite domain $\Omega$, the number of the lattice points is denoted by $|\Omega|$.
Given a sequence $\{x(z)\}$, the partial differences of $x(z)$ are defined by

$$
\begin{gathered}
\nabla_{1} x(z)=x\left(i_{1}+1, i_{2}, \ldots, i_{n}\right)-x\left(i_{1}, i_{2}, \ldots, i_{n}\right), \\
\nabla_{2} x(z)=x\left(i_{1}, i_{2}+1, \ldots, i_{n}\right)-x\left(i_{1}, i_{2}, \ldots, i_{n}\right), \\
\vdots \\
\nabla_{n} x(z)=x\left(i_{1}, i_{2}+1, \ldots, i_{n}\right)-x\left(i_{1}, i_{2}, \ldots, i_{n}\right), \\
\nabla_{1}^{2} x(z)=\nabla_{1}\left(\nabla_{1} x(z)\right), \\
\nabla_{2}^{2} x(z)=\nabla_{2}\left(\nabla_{2} x(z)\right), \ldots, \nabla_{n}^{2} x(z)=\nabla_{n}\left(\nabla_{n} x(z)\right) .
\end{gathered}
$$

The discrete Laplace operator will be denoted by $\Delta$ which is defined by

$$
\begin{aligned}
\Delta x(z)= & \nabla_{1}^{2} x\left(i_{1}-1, i_{2}, \ldots, i_{n}\right) \\
& +\nabla_{2}^{2} x\left(i_{1}, i_{2}-1, \ldots, i_{n}\right)+\cdots \\
& +\nabla_{n}^{2} x\left(i_{1}, i_{2}, \ldots, i_{n}-1\right) .
\end{aligned}
$$


Problem (1) can be regarded as the discrete analogue of the elliptic boundary problem of the form

$$
\begin{aligned}
& -\Delta u=g(x, u), \quad x \in \Phi, \\
& u(x)=0, \quad x \in \partial \Phi,
\end{aligned}
$$

where $\Phi \subset R^{n}$ and $g$ satisfy some conditions and $\Delta$ is the Laplacian operator defined by

$$
\Delta u=\sum_{k=1}^{n} \frac{\partial^{2} u}{\partial x_{i}^{2}} .
$$

There exist many differences between (1) and (4). As a simplest example, let us consider the continuous linear equation:

$$
\begin{aligned}
& -\Delta u=\mu p(x) u, \quad x \in \Phi, \\
& u(x)=0, \quad x \in \partial \Phi
\end{aligned}
$$

It is well known that the operator $-\Delta$ has a sequence of eigenvalues:

$$
0<\mu^{(1)}<\mu^{(2)} \leq \cdots \leq \mu^{(n)} \leq \cdots
$$

when the weight function $p: \Phi \rightarrow R^{+}$is assumed to be in $L^{r}(\Phi)$ with $r>n / 2$ and $p(x)>0$ on a subset of positive measures of $\Phi$. In particular, from Krein-Rutman theorem, the principal eigenvalue $\mu^{(1)}$ is simple, in the sense that the eigenspace is one-dimensional, and the corresponding eigenfunction $\varphi^{(1)}$ can be chosen to be positive. At the same time, we also have $\lim _{n \rightarrow \infty} \mu^{(n)}=\infty$. Note that when $\Omega$ is a finite domain, the discrete problem

$$
\begin{aligned}
-\Delta x(z) & =\mu p(z) x(z), \quad z \in \Omega, \\
x(z) & =0, \quad z \in \partial \Omega
\end{aligned}
$$

is only a finite dimension problem. This will induce some essential differences between (6) and (8); see $[1,2]$ and the listed references. First of all, we need to ask $p \in L^{r}(\Phi)$ for eigenvalue problem (6); however, corresponding eigenvalue problem (8) does not need to add any conditions; secondly, problem (8) has at most $|\Omega|$ eigenvalues; however, the number of eigenvalues of (6) is infinite; thirdly, in general case, the existence of solutions for (4) will be considered in Sobolev space $H_{0}^{1}(\Phi)$ (see [3-6]), but (1) is not necessary, and so forth. Thus, it is necessary to consider the existence of solutions for problem (1).

By using the difference method, we can obtain problem (1); see [7]. In fact, problem (1) can also be obtained by the discrete heat diffusion; see [8]. Recently, the existence of solutions for problem (1) has been extensively established in the rectangle domain; for example, see [8-20] (by using the eigenvalue method [8,9], critical point theory [10-16], fixed point theorems and the degree theory $[17,18]$, and contraction method and monotone method [18]). For the $n$ dimension case, we only see [21-25]. Pao [21-23] considered the existence of numerical solutions for nonlinear elliptic boundary value problems by using the matrix and vector method. However, [21-23] restricted that the coefficient matrix of system needs to be irreducible. In fact, it is difficult to prove that a matrix is irreducible. In $[24,25]$ the authors mostly established the existence of radial positive solutions by using the fixed point theorems and degree theory.

In this paper, we will consider the nonexistence, existence, and uniqueness of positive solutions of (1) by using the maximum principle, eigenvalue method, sub- and supersolution technique, and monotone method. To this end, the elementary theory will be introduced in the next section. The sub- and supersolution technique, the nonexistence, existence, and uniqueness will be considered in Section 3. In the final section, we will give some applications.

Our results are suitable for any $n$-dimension space and the shape of domain $\Omega$ is not restrained.

\section{Preliminaries}

In this section, we hope to obtain some preliminaries results. They are important for establishing our main results. The obtained results seem to be similar to the corresponding continuous elliptic boundary problem; however, their conditions are different. In particular, the methods of proof are also different.

Consider first the eigenvalue problem

$$
\begin{aligned}
-\Delta x(z)+p(z) x(z) & =\lambda x(z), \quad z \in \Omega, \\
x(z) & =0, \quad z \in \partial \Omega,
\end{aligned}
$$

where $p=\{p(z)\}_{z \in \Omega}$ is real and $\Omega \subset Z^{n}$ is a finite domain.

It is well known that the maximum principle is important. Thus, we firstly give a maximum principle.

Lemma 1. Assume that $r=\{r(z)\}_{z \in \Omega}$ is nonnegative and $x=$ $\{x(z)\}_{z \in \bar{\Omega}}$ satisfies the difference inequality

$$
-\Delta x(z)+r(z) x(z) \leq 0(\geq 0) \quad \text { for } z \in \Omega .
$$

Then $x$ cannot achieve a nonnegative maximum (nonpositive minimum) in the interior of $\Omega$ unless it is constant.

Proof. Suppose that there exists $z^{0} \in \Omega$ such that $x\left(z^{0}\right)=$ $M=\max _{z \in \bar{\Omega}} x(z) \geq 0$. Then we have $\Delta x\left(z^{0}\right) \leq 0$. In view of (10), we must have $\Delta x\left(z^{0}\right)=0$. That is,

$$
\begin{aligned}
& x\left(i_{1}^{0}+1, i_{2}^{0}, \ldots, i_{n}^{0}\right)+x\left(i_{1}^{0}-1, i_{2}^{0}, \ldots, i_{n}^{0}\right)+\cdots \\
& \quad+x\left(i_{1}^{0}, i_{2}^{0}, \ldots, i_{n}^{0}+1\right)+x\left(i_{1}^{0}, i_{2}^{0}, \ldots, i_{n}^{0}-1\right) \\
& \quad=2 n x\left(i_{1}^{0}, i_{2}^{0}, \ldots, i_{n}^{0}\right)=2 n M,
\end{aligned}
$$

which implies that

$$
\begin{aligned}
x\left(i_{1}^{0}+1, i_{2}^{0}, \ldots, i_{n}^{0}\right) & =x\left(i_{1}^{0}-1, i_{2}^{0}, \ldots, i_{n}^{0}\right)=\cdots \\
& =x\left(i_{1}^{0}, i_{2}^{0}, \ldots, i_{n}^{0}+1\right) \\
& =x\left(i_{1}^{0}, i_{2}^{0}, \ldots, i_{n}^{0}-1\right)=M .
\end{aligned}
$$


In the following, we will assert that for any point $z^{1} \in \bar{\Omega}$, $x\left(z^{1}\right)=M$. Let $z_{1}=z^{0}, z_{2}, \ldots, z_{m}=z^{1}$ be a chain of points contained in $\bar{\Omega}$. By using (12), we have $x\left(z_{2}\right)=x\left(z_{1}\right)=M$. If $z_{2}=z^{1}$, the proof is complete. Otherwise, we may repeat the above argument to deduce $x\left(z_{3}\right)=M$. By using finite times induction, we can prove that $x\left(z_{m}\right)=x\left(z^{1}\right)=M$. The result for $-\Delta x(z)+r(z) x(z) \geq 0$ follows by replacement of $x$ by $-x$.

In the following, we will consider eigenvalue problem (9).

Lemma 2. Eigenvalue problem (9) has $|\Omega|$ real eigenvalues $\left\{\lambda_{s}\right\}_{s=1}^{|\Omega|}$ which satisfy

$$
\lambda_{1}(p)<\lambda_{2}(p) \leq \cdots \leq \lambda_{|\Omega|}(p)
$$

where $\lambda_{1}(p)$ is simple and the corresponding eigenfunction $\varphi_{1}$ can be chosen to be positive. Moreover, the other eigenfunctions must change sign in $\Omega$.

Proof. Denote the points in $\Omega$ by $z_{1}, z_{2}, \ldots, z_{|\Omega|}$. Let $A=\left(a_{i j}\right)$ be $|\Omega| \times|\Omega|$ matrix, where $a_{i j}=1$ if $z_{i}$ and $z_{j}$ are neighbors and $a_{i j}=0$ otherwise. Then (9) can be written as

$$
(2 n I-A) X+P X=\lambda X,
$$

where $I$ is the $|\Omega| \times|\Omega|$ identity matrix, $X=\left(x\left(z_{1}\right), x\left(z_{2}\right), \ldots\right.$, $\left.x\left(z_{|\Omega|}\right)\right)^{T}$, and $P=\operatorname{diag}\left(p\left(z_{1}\right), p\left(z_{2}\right), \ldots, p\left(z_{|\Omega|}\right)\right)$; see [20]. Clearly, the matrix $2 n I-A$ is positive definite and symmetric. The rest of the proof is the same as Lemma 1 in [26].

A comparison result will be obtained in the following.

Lemma 3. Assume that $p(z) \leq \bar{p}(z)$ for $z \in \Omega$. Then $\lambda_{1}(p) \leq$ $\lambda_{1}(\bar{p})$. Moreover, if there exists $z_{0} \in \Omega$ such that $p\left(z_{0}\right)<\bar{p}\left(z_{0}\right)$, then $\lambda_{1}(p)<\lambda_{1}(\bar{p})$.

Proof. By the representation of the first eigenvalue

$$
\lambda_{1}(p)=\inf _{\substack{\|x\|=\left.1 \\ x\right|_{\partial \Omega}=0}} \sum\left(-x \Delta x+p x^{2}\right)
$$

we see that $\lambda_{1}(p) \leq \lambda_{1}(\bar{p})$ for $p(z) \leq \bar{p}(z)$. In view of Lemma 2, let $x_{1}>0$ with $\left\|x_{1}\right\|=1$ being the eigenfunction corresponding to $\lambda_{1}(\bar{p})$; then we have

$$
\begin{aligned}
\lambda_{1}(\bar{p}) & =\sum\left(-x_{1} \Delta x_{1}+\bar{p} x_{1}^{2}\right)>\sum\left(-x_{1} \Delta x_{1}+p x_{1}^{2}\right) \\
& \geq \inf _{\substack{\|x\|=\left.1 \\
x\right|_{\partial \Omega}=0}} \sum\left(-x \Delta x+p x^{2}\right)=\lambda_{1}(p)
\end{aligned}
$$

for $p(z) \leq \bar{p}(z)$ and $p(z) \not \equiv \bar{p}(z)$.

Now, we consider the principal eigenvalue and the corresponding principal function or the corresponding principal vector.
Lemma 4. Let $\lambda_{1}(p)>0$. Then, for any sequence $\{g(z)\}_{z \in \Omega}$, the discrete Dirichlet boundary value problem

$$
\begin{aligned}
-\Delta x(z)+p(z) x(z) & =g(z), \quad z \in \Omega, \\
x(z) & =0, \quad z \in \partial \Omega,
\end{aligned}
$$

has a unique solution. Moreover, if $\{p(z)\}_{z \in \Omega}$ is nonnegative and $\{g(z)\}_{z \in \Omega}$ is nonnegative and not identically zero, the solution is positive.

Proof. Note that problem (17) can be written in matrix and vector form

$$
(2 n I-A) X+P X=G,
$$

where $G=\left(g\left(z_{1}\right), g\left(z_{2}\right), \ldots, g\left(z_{|\Omega|}\right)\right)^{T}$ and $A, I$, and $P$ are as in (14). Since 0 is not an eigenvalue of (17), $(2 n I-A)+P$ is reversible. Hence, problem (17) has a unique solution. The positivity of the solution is a direct result of Lemma 1.

Consider now the eigenvalue problem

$$
\begin{aligned}
-\Delta x(z) & =\lambda q(z) x(z), \quad z \in \Omega, \\
x(z) & =0, \quad z \in \partial \Omega,
\end{aligned}
$$

where $q=\{q(z)\}_{z \in \Omega}$ is nonnegative and not identically zero in $\Omega$ and $\Omega \subset Z^{n}$ is a finite domain.

For eigenvalue problem (19), we have the following result.

Lemma 5. The first eigenvalue $\lambda^{(1)}(q)$ of problem (19) is simple and the corresponding eigenfunction $\varphi^{(1)}$ can be chosen to be positive. In particular, if $\alpha$ is a positive constant, then

$$
\lambda^{(1)}(\alpha q)=\frac{1}{\alpha} \lambda^{(1)}(q)
$$

Proof. Consider the eigenvalue problem

$$
\begin{aligned}
-\Delta x(z)-\lambda^{(1)}(q) q(z) x(\mathrm{z}) & =\mu x(z), \quad z \in \Omega, \\
x(z) & =0, \quad z \in \partial \Omega .
\end{aligned}
$$

It is easy to see that $\lambda^{(1)}(q)$ is the first eigenvalue for (19) with corresponding eigenfunction $\varphi^{(1)}$ if and only if 0 is the first eigenvalue of (21) with corresponding eigenfunction $\varphi^{(1)}$. In view of Lemma 2, we complete the proof.

Lemma 6. Let $q$ satisfy the condition of (19) and $\lambda<$ $\lambda^{(1)}(q)$. Then for any sequence $\{g(z)\}_{z \in \Omega}$, the discrete Dirichlet boundary value problem

$$
\begin{aligned}
-\Delta x(z) & =\lambda q(z) x(z)+g(z), \quad z \in \Omega, \\
x(z) & =0, \quad z \in \partial \Omega,
\end{aligned}
$$

has a unique solution. Moreover, if $\{g(z)\}_{z \in \Omega}$ is nonnegative and not identically zero, then the solution is positive. 
Proof. Problem (22) can be written in matrix and vector form

$$
(2 n I-A) X=\lambda Q X+G
$$

where $G=\left(g\left(z_{1}\right), g\left(z_{2}\right), \ldots, g\left(z_{|\Omega|}\right)\right)^{T}, Q=\operatorname{diag}\left(q\left(z_{1}\right), q\left(z_{2}\right)\right.$, $\left.\ldots, q\left(z_{|\Omega|}\right)\right)$, and $A$ and $I$ are as in (14). As Lemma 4, problem (22) has a unique solution for any $\lambda<\lambda^{(1)}(q)$. If $\lambda \leq 0$, the positivity of the solution is a direct result of Lemma 1 . In the following, we assume that $0<\lambda<\lambda^{(1)}(q)$. Assume without loss of generality that $|q(z)|<1$ for $z \in \Omega$. Write (23) in the form

$$
((2 n+\lambda) I-A) X=\lambda(Q+I) X+G
$$

For any $\lambda>0$, let $\mu_{1, \lambda}>0$ denote the first eigenvalue of

$$
((2 n+\lambda) I-A) X=\mu(Q+I) X .
$$

We claim that $0<\lambda<\lambda^{(1)}(q)$ implies that $\lambda<\mu_{1, \lambda}$. Indeed, suppose by contradiction that $\lambda \geq \mu_{1, \lambda}$. Then

$$
\begin{aligned}
\frac{1}{\mu_{1, \lambda}} & =\sup \frac{X^{T}(Q+I) X}{X^{T}((2 n+\lambda) I-A) X} \\
& \leq \sup \frac{X^{T}(Q+I) X}{X^{T}\left(\left(2 n+\mu_{1, \lambda}\right) I-A\right) X} .
\end{aligned}
$$

Hence, for any $\epsilon>0$ there exists $X$ such that

$$
\frac{1}{\mu_{1, \lambda}}-\epsilon \leq \frac{X^{T}(Q+I) X}{X^{T}\left(\left(2 n+\mu_{1, \lambda}\right) I-A\right) X}
$$

or equivalently

$$
\frac{1}{\mu_{1, \lambda}}-\epsilon-\mu_{1, \lambda} \epsilon \frac{\|X\|^{2}}{X^{T}(2 n I-A) X} \leq \frac{X^{T} Q X}{X^{T}(2 n I-A) X} .
$$

By the representation of the first eigenvalue we get

$$
\frac{1}{\mu_{1, \lambda}}-\epsilon-\mu_{1, \lambda} \epsilon \frac{1}{\lambda^{(1)}(1)} \leq \frac{1}{\lambda^{(1)}(q)}, \quad \forall \epsilon>0 .
$$

Let $\epsilon \rightarrow 0$, we have

$$
\lambda^{(1)}(q) \leq \mu_{1, \lambda} \leq \lambda<\lambda^{(1)}(q) .
$$

That is contradiction. Thus $\lambda<\mu_{1, \lambda}$. Write (24) in the form

$$
\begin{aligned}
X= & \lambda((2 n+\lambda) I-A)^{-1}(Q+I) X \\
& +((2 n+\lambda) I-A)^{-1} G .
\end{aligned}
$$

Denote $M=\lambda((2 n+\lambda) I-A)^{-1}(Q+I)$. Since $\lambda<\mu_{1, \lambda}$, the largest eigenvalue of $M$ is less than 1 . Hence

$$
X=(I-M)^{-1}((2 n+\lambda) I-A)^{-1} G
$$

and the positivity of $X$ follows from the positivity of $M$ and $((2 n+\lambda) I-A)^{-1}$.

\section{Nonexistence, Existence, and Uniqueness of Positive Solutions}

First of all, we give a nonexistence result.

Theorem 7. Assume that

$$
\sup _{x \neq 0} \frac{\sum_{z \in \Omega} x f(z, x)}{\|x\|^{2}}<\lambda_{1}(0)
$$

or

$$
\inf _{x \neq 0} \frac{\sum_{z \in \Omega} x f(z, x)}{\|x\|^{2}}>\lambda_{|\Omega|}(0)
$$

holds. Then problem (1) has no nonzero solution.

Proof. By contradiction, suppose that $x$ is a nonzero solution of (1); then we have

$$
-\sum_{z \in \Omega} x(z) \Delta x(z)=\sum_{z \in \Omega} x(z) f(z, x(z))
$$

or

$$
\frac{-\sum_{z \in \Omega} x(z) \Delta x(z)}{\|x\|^{2}}=\frac{\sum_{z \in \Omega} x(z) f(z, x(z))}{\|x\|^{2}},
$$

where

$$
\|x\|=\sqrt{\sum_{z \in \Omega} x^{2}(z)} .
$$

In view of [27], we know that

$$
\begin{aligned}
& \max _{x \neq 0} \frac{-\sum_{z \in \Omega} x(z) \Delta x(z)}{\|x\|^{2}}=\lambda_{|\Omega|}(0), \\
& \min _{x \neq 0} \frac{-\sum_{z \in \Omega} x(z) \Delta x(z)}{\|x\|^{2}}=\lambda_{1}(0) .
\end{aligned}
$$

In view of (36) and (38), we complete the proof.

Definition 8. A sequence $x=\{x(z)\}_{z \in \bar{\Omega}}$ is said to be a supersolution (subsolution) of (1) if

$$
\begin{aligned}
-\Delta x(z) & \geq(\leq) f(z, x(z)), \quad z \in \Omega, \\
x(z) & \geq(\leq) 0, \quad z \in \partial \Omega .
\end{aligned}
$$

Theorem 9. Suppose that problem (1) has a subsolution $\underline{x}$ and a supersolution $\bar{x}$, with $\underline{x} \leq \bar{x}$. Assume that $f(z, s)$ is continuous with $s \in[a, b]$ and there exists a constant $k \geq 0$ such that

$$
f\left(z, s_{2}\right)-f\left(z, s_{1}\right) \geq-k\left(s_{2}-s_{1}\right)
$$

for all $z \in \Omega$ and $s_{2} \geq s_{1}$ with $s_{1}, s_{2} \in[a, b]$ (where $a=\min _{z \in \Omega} \underline{x}(z)$ and $b=\max _{z \in \Omega} \bar{x}(z)$ ). Then problem (1) has solutions $u$ and $v$ such that $\underline{x} \leq u \leq v \leq \bar{x}$. Moreover any solution $x$ of (1) with $\underline{x} \leq x \leq \bar{x}$ satisfies $u \leq x \leq v$. 
Proof. In view of Lemma 4 , for any sequence $u=\{u(z)\}_{z \in \Omega}$, the problem

$$
\begin{aligned}
-\Delta w(z)+k w(z) & =f(z, u(z))+k u(z), \quad z \in \Omega, \\
w(z) & =0, \quad z \in \partial \Omega,
\end{aligned}
$$

has a unique solution. This defines a mapping $T: u \rightarrow w$ which is monotone in $[\underline{x}, \bar{x}]$; that is, if $\underline{x} \leq u_{1} \leq u_{2} \leq \bar{x}$, then $w_{1}=T u_{1} \leq T u_{2}=w_{2}$. Indeed, writing (41) with $u=u_{1}$ and $u=u_{2}$ and subtracting one from the other, we obtain

$$
\begin{aligned}
-\Delta & \left(w_{2}(z)-w_{1}(z)\right)+k\left(w_{2}(z)-w_{1}(z)\right) \\
= & f\left(z, u_{2}(z)\right)-f\left(z, u_{1}(z)\right) \\
& +k\left(u_{2}(z)-u_{1}(z)\right) .
\end{aligned}
$$

By using (40) and the maximum principle, we can obtain $T u_{1} \leq T u_{2}$.

Let $u_{k}=T u_{k-1}, u_{0}=\underline{x} ; v_{k}=T v_{k-1}, v_{0}=\bar{x}$. In the following, we claim that

$$
\underline{x}=u_{0} \leq u_{1} \leq u_{2} \leq \cdots \leq v_{2} \leq v_{1} \leq v_{0}=\bar{x}, \quad \text { in } \Omega .
$$

First of all, by using (41) and the definition of subsolution, we see that

$$
\begin{gathered}
-\Delta\left(u_{0}(z)-u_{1}(z)\right)+k\left(u_{0}(z)-u_{1}(z)\right) \\
=-\Delta u_{0}(z)+k u_{0}(z)-\left[f\left(z, u_{0}(z)\right)+k u_{0}(z)\right] \\
=-\Delta \underline{x}(z)-f(z, \underline{x}(z)) \leq 0 \quad \text { for } z \in \Omega, \\
u_{0}(z)-u_{1}(z)=\underline{x}(z) \leq 0 \quad \text { for } z \in \partial \Omega .
\end{gathered}
$$

This implies by the maximum principle that $u_{0}(z)-u_{1}(z) \leq 0$ in $\bar{\Omega}$. A similar argument gives $v_{1} \leq v_{0}$. The monotonicity of $T$ gives the rest. So there exist $u$ and $v$ such that

$$
\begin{aligned}
& \lim _{k \rightarrow \infty} u_{k}=u, \\
& \lim _{k \rightarrow \infty} v_{k}=v .
\end{aligned}
$$

The proof is complete.

Remark 10. For the corresponding partial differential equations, $f$ need to be $C^{\alpha}(0<\alpha<1)$ function; see [3].

In the following, we will give an existence result. To this end, we assume that $f(z, x)$ is continuous with $x$ and satisfies the following three conditions:

$\left(\mathrm{H}_{1}\right)$ there exists a nonnegative and nontrivial sequence $f_{0}=\left\{f_{0}(z)\right\}_{z \in \Omega}$ and a positive number $s_{0}$ such that

$$
f(z, x) \geq f_{0}(z) x \text { for } 0<x<s_{0}, z \in \Omega \text {; }
$$

$\left(\mathrm{H}_{2}\right)$ there exist nonnegative sequences $f_{\infty}=\left\{f_{\infty}(z)\right\}_{z \in \Omega}$ and $c=\{c(z)\}_{z \in \Omega}$ such that

$$
f(z, x) \leq f_{\infty}(z) x+c(z) \quad \text { for } x \geq 0, z \in \Omega
$$

$\left(\mathrm{H}_{3}\right)$ for any real number $M>0$, there exists a constant $k \geq$ 0 such that $f(z, x)+k x$ is nondecreasing for $|x| \leq M$.

Theorem 11. Assume that conditions $\left(\mathrm{H}_{1}\right),\left(\mathrm{H}_{2}\right)$, and $\left(\mathrm{H}_{3}\right)$ hold. Suppose that $\lambda^{(1)}\left(f_{0}(z)\right)<1$ and $\lambda^{(1)}\left(f_{\infty}(z)\right)>1$. Then Dirichlet problem (1) has a positive solution.

Proof. Note that $\lambda^{(1)}\left(f_{\infty}(z)\right)>1$. In view of Lemma 6 , the Dirichlet problem

$$
\begin{aligned}
& -\Delta w=f_{\infty}(z) w(z)+c(z), \quad z \in \Omega \\
& w(z)=0, \quad z \in \partial \Omega
\end{aligned}
$$

has a unique positive solution. It is a supersolution of (1) and any nonnegative solution $x$ of (1) satisfies $x \leq w$. On the other hand, the eigenvalue problem

$$
\begin{aligned}
& -\Delta w=\lambda f_{0}(z) w(z), \quad z \in \Omega, \\
& w(z)=0, \quad z \in \partial \Omega,
\end{aligned}
$$

has the first eigenvalue $\lambda^{(1)}\left(f_{0}(z)\right)<1$ and the corresponding positive eigenfunction $\varphi^{(1)}(z)$. Choose an enough small positive number $\varepsilon$ such that $\varepsilon \varphi_{1}$ is a subsolution of (1). In view of Theorem 9 and condition $\left(\mathrm{H}_{3}\right)$, problem (1) has a positive solution.

Remark 12. For the partial differential equations, the corresponding result has been obtained in [28] when $f \in C^{\alpha}$.

Theorem 13. Assume that the hypotheses of Theorem 11 hold. Suppose that the function $f(z, x) / x$ is strictly monotone for $x>$ 0 . Then problem (1) has a unique positive solution.

Proof. Assume that $x_{1}$ and $x_{2}$ are two distinct positive solutions. If neither $x_{1} \geq x_{2}$ nor $x_{1} \leq x_{2}$, by using Theorem 9 we can obtain a positive solution $w$ of (1) such that $w \geq x_{1}$ and $w \geq x_{2}$. Thus we may assume without loss of generality that $x_{1} \leq x_{2}$ and $x_{1} \neq \equiv x_{2}$. Then, for $i=1,2$ we have

$$
\begin{aligned}
& -\Delta x_{i}=\frac{f\left(z, x_{i}\right)}{x_{i}} x_{i}, \quad z \in \Omega, \\
& x_{i}(z)=0, \quad z \in \partial \Omega .
\end{aligned}
$$

Note that the function $f(z, x) / x$ is strictly monotone for $x>0$, which implies that $f\left(z, x_{1}\right) / x_{1}>f\left(z, x_{2}\right) / x_{2}$ or $f\left(z, x_{1}\right) / x_{1}<f\left(z, x_{2}\right) / x_{2}$. Thus, by Lemma 3 , we have

$$
\lambda_{1}\left(-\frac{f\left(z, x_{1}\right)}{x_{1}}\right)<\lambda_{1}\left(-\frac{f\left(z, x_{2}\right)}{x_{2}}\right)
$$

or

$$
\lambda_{1}\left(-\frac{f\left(z, x_{1}\right)}{x_{1}}\right)>\lambda_{1}\left(-\frac{f\left(z, x_{2}\right)}{x_{2}}\right) .
$$

However, from (50) and the fact that $x_{i}>0$ in $\Omega$ we arrive at the contradictory statement that $\lambda_{1}\left(-f\left(z, x_{1}\right) / x_{1}\right)=$ $\lambda_{1}\left(-f\left(z, x_{2}\right) / x_{2}\right)=0$. The proof is complete. 
Remark 14. For the corresponding partial differential equations, the conditions of Theorem 13 need to add that the function $f(z, x) / x$ is uniformly continuous for $0<x \leq x_{0}$ for some $x_{0}>0$ or that there exist constants $c>0, x_{0}>0$, and $0<\alpha<2 / n$ such that $f(z, x) / x \leq c x^{-\alpha}$ for $0<x<x_{0}$. See Theorems 2.4 and 2.5 in [4].

Theorem 15. Let $f(z, x)=x h(z, x)$. Suppose that there exists a constant $M>0$ such that $h(z, x) \leq 0$ for $z \in \Omega$ and $x \geq M$ and that $h(z, x)$ is smooth and strictly decreasing in $x \in[0, M]$. Then problem (1) admits at most one positive solution.

Proof. By the maximum principle and the hypothesis, any solution $x$ of problem (1) satisfies $x \leq M$ in $\Omega$. Assume that $x_{1}$ and $x_{2}$ are two distinct positive solutions. Under the hypothesis, 0 and $M$ are sub- and suppersolution of (1). If neither $x_{1} \geq x_{2}$ nor $x_{1} \leq x_{2}$, by Theorem 9 , there exists a maximal solution $v$ by using $M$. Hence, $x_{1} \leq v$ and $x_{2} \leq v$. Thus we may assume without loss of generality that $x_{1} \leq x_{2}$ and $x_{1} \not \equiv x_{2}$. Then, we have

$$
\begin{aligned}
& -\Delta x_{i}=h\left(z, x_{i}\right) x_{i}, \quad z \in \Omega, \\
& x_{i}(z)=0, \quad z \in \partial \Omega .
\end{aligned}
$$

Hence, $\lambda_{1}\left(-h\left(z, x_{1}\right)\right)=\lambda_{1}\left(-h\left(z, x_{2}\right)\right)=0$. On the other hand, by the assumption on $h$, we have

$$
\lambda_{1}\left(-h\left(z, x_{1}\right)\right)<\lambda_{1}\left(-h\left(z, x_{2}\right)\right) .
$$

That is a contradiction.

Remark 16. The result of corresponding partial differential equations has been obtained in [5].

\section{Applications}

Consider first the discrete Dirichlet boundary value problem

$$
\begin{aligned}
-\Delta x(z) & =\lambda q(z) x(z)(1-x(z)), \quad z \in \Omega, \\
x(z) & =0, \quad z \in \partial \Omega,
\end{aligned}
$$

where $q$ and $\Omega$ are as in (19); moreover, $q$ is bounded.

By the maximum principle, any nonnegative and nontrivial solution $x$ of (55) must satisfy $0<x<1$. For any $s_{1}, s_{2} \in[0,1]$ with $s_{2} \geq s_{1}$, we have

$$
\begin{aligned}
& \lambda s_{2}\left(1-s_{2}\right)-\lambda s_{1}\left(1-s_{1}\right)=\lambda\left(s_{2}-s_{1}\right)\left(1-s_{1}-s_{2}\right) \\
& \quad \geq-\lambda\left(s_{2}-s_{1}\right) .
\end{aligned}
$$

Let $k=\lambda \max _{z \in \Omega} p(z)$, then condition (40) holds. Assume that $\lambda^{(1)}(q)$ is the first eigenvalue of (19) and $\varphi^{(1)}$ is the corresponding positive eigenfunction with $\left\|\varphi^{(1)}\right\|=1$. For $\lambda>\lambda^{(1)}(q)$, we can choose an enough small positive number $\varepsilon$ such that $\varepsilon \varphi^{(1)}$ is a subsolution of (55). On the other hand, $\bar{x}(z) \equiv 1$ for $z \in \bar{\Omega}$ is a supersolution of (55). In view of Theorem 9, problem (55) has a positive solution. Since the function $f(z, u)=\lambda q(z) u(1-u)$ satisfies all conditions of Theorem 15, the positive solution is unique. Besides, in view of Theorem 7, we can immediately obtain the nonexistence result. Thus, we obtain the following theorem.
Theorem 17. When $\lambda>\lambda^{(1)}(q)$, problem (55) has a unique positive solution $x(z)$. Moreover, $0<x(z)<1$ for $z \in \Omega$. When $\lambda \leq \lambda^{(1)}(q)$, problem (55) has no nontrivial and nonnegative solutions.

Remark 18. When $n=1$ and $q(z) \equiv 1$, Theorem 17 is the main result in [19]. However, the methods of proof are different.

Now consider the discrete Dirichlet boundary value problems

$$
\begin{aligned}
-\Delta x(z) & =\lambda e^{-x(z)}, \quad z \in \Omega, \\
x(z) & =0, \quad z \in \partial \Omega, \\
-\Delta x(z) & =\lambda x^{\alpha}(z), \quad z \in \Omega, \\
x(z) & =0, \quad z \in \partial \Omega,
\end{aligned}
$$

where $0<\alpha<1$. For any $\lambda>0$, we have the following:

(a) $\lim _{x \rightarrow 0+0}\left(\lambda e^{-x} / x\right)=+\infty, \lim _{x \rightarrow 0+0}\left(\lambda x^{\alpha} / x\right)=+\infty$;

(b) $\lambda e^{-x} \leq \epsilon x+\lambda, \lambda x^{\alpha} \leq \epsilon x+M$ for $x \geq 0$, where $\epsilon$ is an arbitrarily small positive constant and $M=M(\epsilon)$ is a constant large enough;

(c) $\lambda e^{-x}+\lambda x$ and $\lambda x^{\alpha}$ are increasing for $x \geq 0$;

(d) $\lambda e^{-x} / x$ and $\lambda x^{\alpha} / x$ are strictly decreasing for $x \geq 0$.

In view of Theorem 13, we can immediately obtain the following result.

Theorem 19. For any $\lambda>0$, problems (57) and (58) have a unique positive solution.

Remark 20. When $\lambda=1$, the corresponding continuous result of (58) has been obtained in any smooth bounded domain [6].

\section{Conflict of Interests}

The authors declare that there is no conflict of interests regarding the publication of this paper.

\section{Acknowledgment}

This work is supported by the National Natural Science Foundation of China (no. 11371277).

\section{References}

[1] S. Flach and C. R. Willis, "Discrete breathers," Physics Reports, vol. 295, no. 5, pp. 181-264, 1998.

[2] S. Flach and A. V. Gorbach, "Discrete breathers-advances in theory and applications," Physics Reports, vol. 467, no. 1-3, pp. $1-116,2008$.

[3] H. Amann, "On the existence of positive solutions of nonlinear elliptic boundary value problems," Indiana University Mathematics Journal, vol. 21, pp. 125-146, 1971.

[4] D. D. Figueiredo, "Positive solutions of semilinear elliptic problems," in Differential Equations, vol. 957 of Lecture Notes in Mathematics, pp. 34-87, Springer, Berlin, Germany, 1982. 
[5] P. Hess, "On uniqueness of positive solutions of nonlinear elliptic boundary value problem," Mathematische Zeitschrift, vol. 154 , no. 1, pp. 17-18, 1977.

[6] H. Brezis and S. Kamin, "Sublinear elliptic equation in $\mathbb{R}^{\mathrm{n}}$," Manuscripta Mathematica, vol. 74, no. 1, pp. 87-106, 1992.

[7] G. I. Marchuk, Methods of Numerical Mathematics, Springer, New York, NY, USA, 2nd edition, 1982.

[8] S. S. Cheng, Partial Difference Equations, Taylor \& Francis, London, UK, 2003.

[9] T. Fort, "The loaded vibrating net and resulting boundary-value problems for a partial difference equation of the second order," Journal of Mathematics and Physics, vol. 33, pp. 94-104, 1954.

[10] G. Zhang and S. S. Cheng, "Existence of solutions for a nonlinear system with a parameter," Journal of Mathematical Analysis and Applications, vol. 314, no. 1, pp. 311-319, 2006.

[11] G. Zhang, "Existence of nontrivial solutions for discrete elliptic boundary value problems," Numerical Methods for Partial Differential Equations, vol. 22, no. 6, pp. 1479-1488, 2006.

[12] G. Zhang, "Existence of non-zero solutions for a nonlinear system with a parameter," Nonlinear Analysis: Theory, Methods \& Applications, vol. 66, no. 6, pp. 1410-1416, 2007.

[13] H. S. Tang, W. Luo, X. Li, and M. J. Ma, "Nontrivial solutions of discrete elliptic boundary value problems," Computers \& Mathematics with Applications, vol. 55, no. 8, pp. 1854-1860, 2008.

[14] P. Candito and G. M. Bisci, "Existence of solutions for a nonlinear algebraic system with a parameter," Applied Mathematics and Computation, vol. 218, no. 23, pp. 11700-11707, 2012.

[15] G. M. Bisci and D. Repovš, "Nonlinear algebraic systems with discontinuous terms," Journal of Mathematical Analysis and Applications, vol. 398, no. 2, pp. 846-856, 2013.

[16] M. Galewski, "On the existence of solutions for a system of difference equations with non-monotone nonlinearity," Applied Mathematics Letters, vol. 24, no. 12, pp. 2015-2018, 2011.

[17] G. Zhang and W. Feng, "On the number of positive solutions of a nonlinear algebraic system," Linear Algebra and Its Applications, vol. 422, no. 2-3, pp. 404-421, 2007.

[18] W. Feng and G. Zhang, "Eigenvalue and spectral intervals for a nonlinear algebraic system," Linear Algebra and Its Applications, vol. 439, no. 1, pp. 1-20, 2013.

[19] S. S. Cheng and F.-Y. Hsieh, "Existence and localization theorems for a discrete nonlinear eigenvalue problem," Mathematical and Computer Modelling, vol. 34, no. 5-6, pp. 623-640, 2001.

[20] S. S. Cheng and S. S. Lin, "Existence and uniqueness theorems for nonlinear difference boundary value problems," Utilitas Mathematica, vol. 39, pp. 167-186, 1991.

[21] C. V. Pao, "Block monotone iterative methods for numerical solutions of nonlinear elliptic equations," Numerische Mathematik, vol. 72, no. 2, pp. 239-262, 1995.

[22] C. V. Pao, "Accelerated monotone iterations for numerical solutions of nonlinear elliptic boundary value problems," Computers \& Mathematics with Applications, vol. 46, no. 10-11, pp. 15351544, 2003.

[23] C. V. Pao, "Monotone iterative methods for numerical solutions of nonlinear integro-elliptic boundary problems," Applied Mathematics and Computation, vol. 186, no. 2, pp. 1624-1642, 2007.

[24] P. J. Y. Wong and R. P. Agarwal, "Multiple solutions of difference and partial difference equations with Lidstone conditions: boundary value problems and related topics," Mathematical and Computer Modelling, vol. 32, no. 5-6, pp. 699-725, 2000.
[25] P. J. Y. Wong and L. H. Xie, "Three symmetric solutions of Lidstone boundary value problems for difference and partial difference equations: advances in difference equations IV," Computers \& Mathematics with Applications, vol. 45, no. 6-9, pp. 1445-1460, 2003.

[26] X. Li, G. Zhang, and Y. Wang, "Existence and uniqueness of positive solitons for a second-order difference equation," Discrete Dynamics in Nature and Society, vol. 2014, Article ID 503496, 8 pages, 2014.

[27] P. Lancaster and M. Tismenetsky, Theory of Matrices, Academic Press, New York, NY, USA, 1985.

[28] H. Amann, "Fixed point equations and nonlinear eigenvalue problems in ordered Banach spaces," SIAM Review, vol. 18, no. 4, pp. 620-709, 1976. 


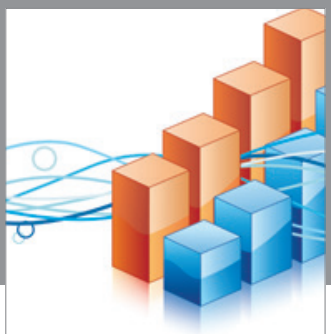

Advances in

Operations Research

vatem alat4

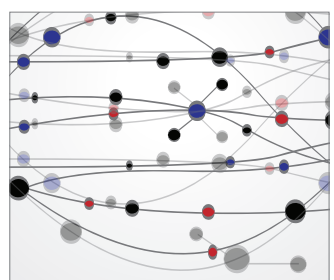

\section{The Scientific} World Journal
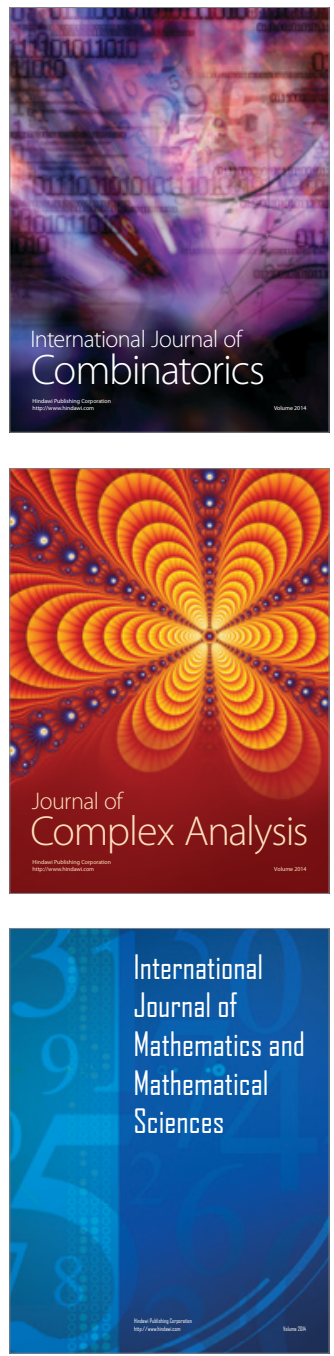
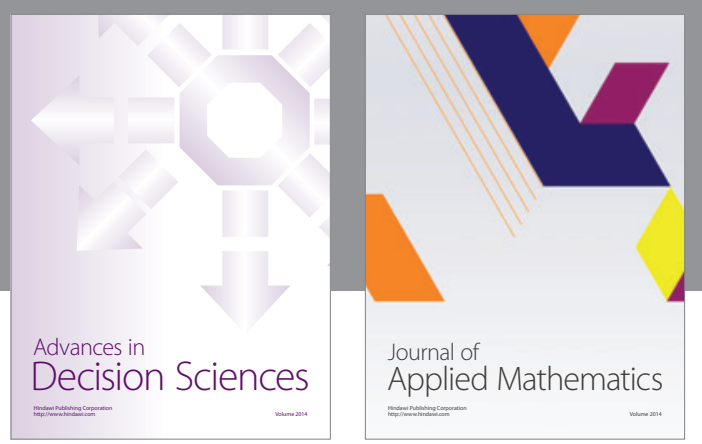

Algebra

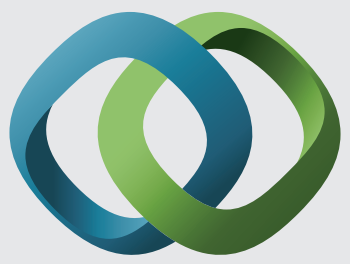

\section{Hindawi}

Submit your manuscripts at

http://www.hindawi.com
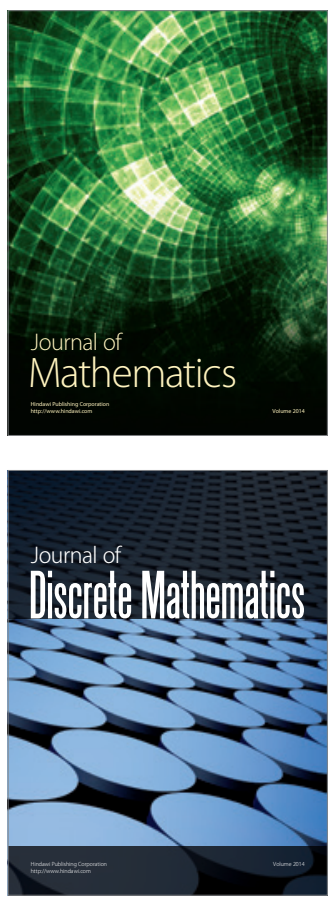

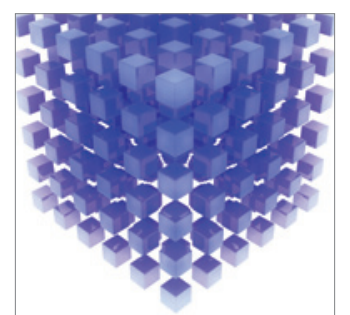

Mathematical Problems in Engineering
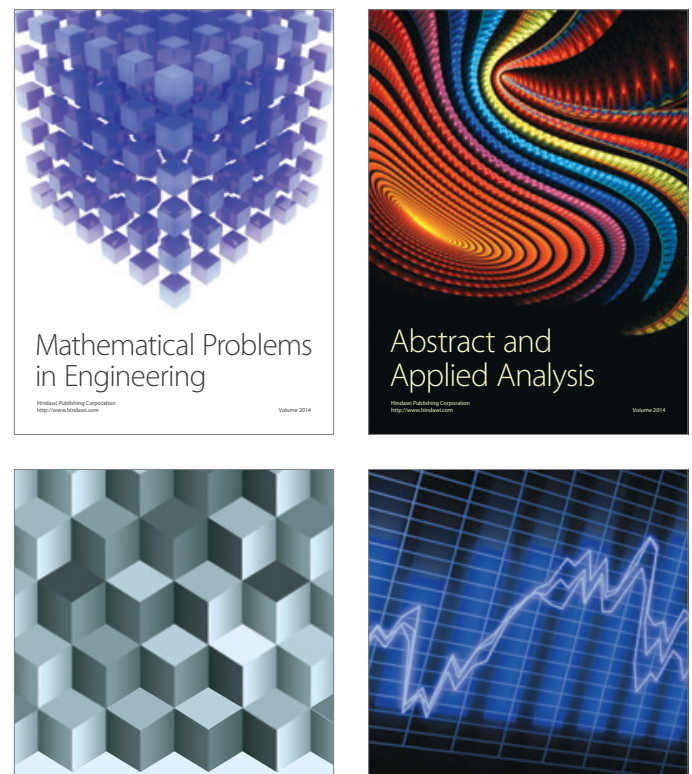

Journal of

Function Spaces

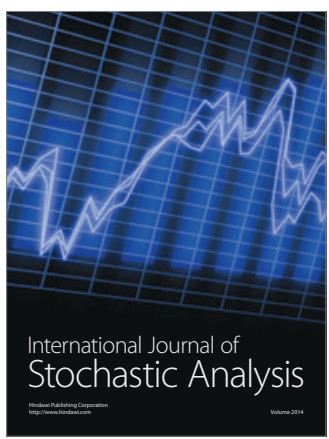

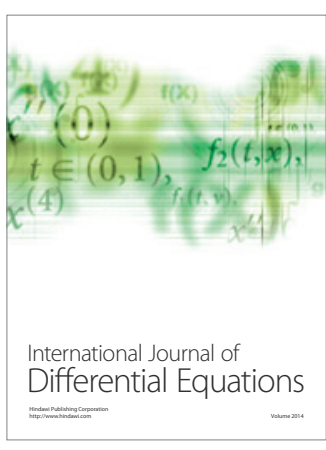
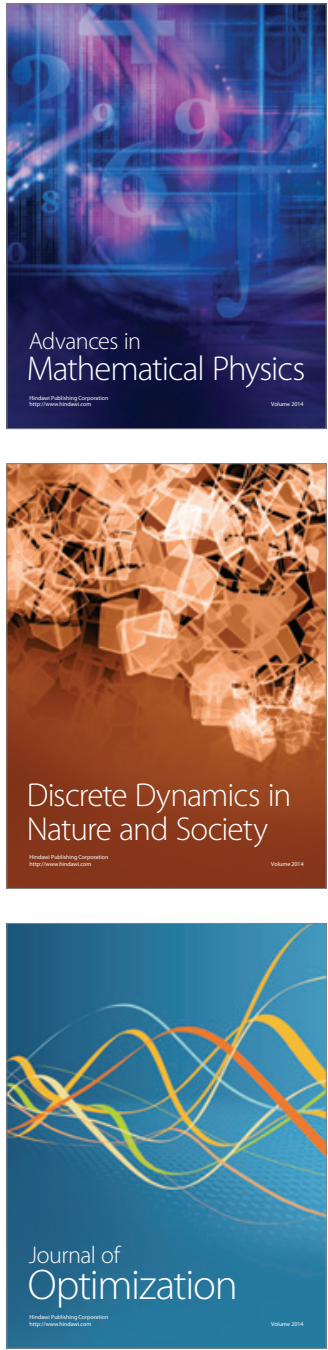